\title{
Improving the Thermal Properties of Polycarbonate via the Copolymerization of a Small Amount of Bisphenol Fluorene with Bisphenol A
}

\author{
Xiaozhou Zhang $\mathbb{D}^{1},{ }^{1}$ Yang Liu, ${ }^{1}$ Xin Li, ${ }^{1}$ Xin Liu, ${ }^{1}$ Xigao Jian, ${ }^{2}$ and Jinyan Wang ${ }^{2}$ \\ ${ }^{1}$ Key Laboratory of Polymer Matrix Composites, Heilongjiang Province, College of Materials Science and Engineering, \\ Qiqihar University, Wenhua Street 42, Qiqihar, Heilongjiang 161006, China \\ ${ }^{2}$ College of Chemical Industry, Dalian University of Technology, Linggong Road 2, Dalian, Liaoning 116024, China
}

Correspondence should be addressed to Jinyan Wang; wangjinyan@dlut.edu.cn

Received 1 November 2021; Revised 10 January 2022; Accepted 11 January 2022; Published 1 February 2022

Academic Editor: Peter Foot

Copyright (c) 2022 Xiaozhou Zhang et al. This is an open access article distributed under the Creative Commons Attribution License, which permits unrestricted use, distribution, and reproduction in any medium, provided the original work is properly cited.

\begin{abstract}
Polycarbonate is an attractive transparent plastic with high mechanical/thermal properties. A family of copolycarbonates of bisphenol-A (BPA), 9, 9-bis (4-hydroxyphenyl) fluorene (BHPF), and diphenyl carbonate (DPC) were prepared by a transesterification polymerization. The weight-average molecular weight of the polycarbonates ranges from 65,000 to $107,000 \mathrm{~g} /$ mol; the copolycarbonates showed $T_{g}$ and $T_{d-5 \%}$ from $63-70^{\circ} \mathrm{C}$ and $100-105^{\circ} \mathrm{C}$ higher than the control, respectively. Meanwhile, the processing properties of polycarbonate remain unchanged. These properties endow the polymers with potential for use as high-temperature resistance materials.
\end{abstract}

\section{Introduction}

Polycarbonate is an attractive transparent plastic with good optical transparency, excellent impact resistance, and high tensile strength [1-5]; thus, typical polycarbonate such as bisphenol A polycarbonate (APC) is widely used in food industry and building, automotive, aircraft, data storage, electrical, and communications hardware [6-10].

At present, the total capacity of APC in the world has reached 5.465 million tons, but most APCs are produced in the process of phosgene formed by $\mathrm{CO}$ and chlorine, which is a toxic and not friendly to environment. Based on the concept of sustainable chemistry, $\mathrm{CO}_{2}$ is used to partially replace the traditional toxic compounds such as $\mathrm{CO}$ and phosgene for the synthesis of cyclic carbonates [11-13] and polycarbonate $[14,15]$. Fukuoka et al. [16] used $\mathrm{CO}_{2}$ and ethylene oxide to synthesize DPC and then produced PC with BPA, so as to avoid using phosgene. Therefore, using DPC and BPA to synthesize polycarbonate gains more attention recently.

In addition, considering the application of APC in aerospace and other special fields, the heat resistance of APC has to be enhanced to meet the requirement of severe applied environments. The introduction of aromatic macromolecules to improve the chain segment rigidity and glass transition temperature is an important way to regulate and improve the heat resistance of APC. Fluorene ring was frequently used to improve the heat resistance of polycarbonates. Compared with BPA, the two phenol groups in 9, 9double (4-hydroxyphenyl) fluorene molecular are connected to a unique structure of fluorene ring, namely, Cardo ring, instead of straight chain propane groups, and the rigidity of the ring structure is significantly higher than that of the nonring structure $[17,18]$. At the same time, as four benzene rings attach to a carbon, BHPF has good thermal stability and chemical stability. In addition, the molecules of these two compounds contain two active hydroxyl groups distributed symmetrically, which is able to polycondense with corresponding compounds to form polymer chains. The introduction of this monomer structure can effectively improve the heat resistance of the polymer and obtain better optical and molding properties [19]. Such improvements of polymers in transparency, heat resistance, and insulation 


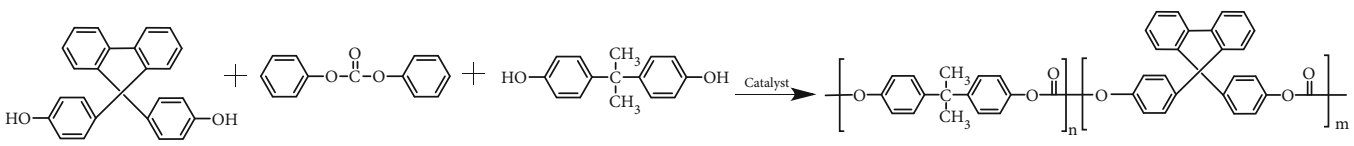

Scheme 1: Reaction equation of PC from BPA, BHPF, and DPC.

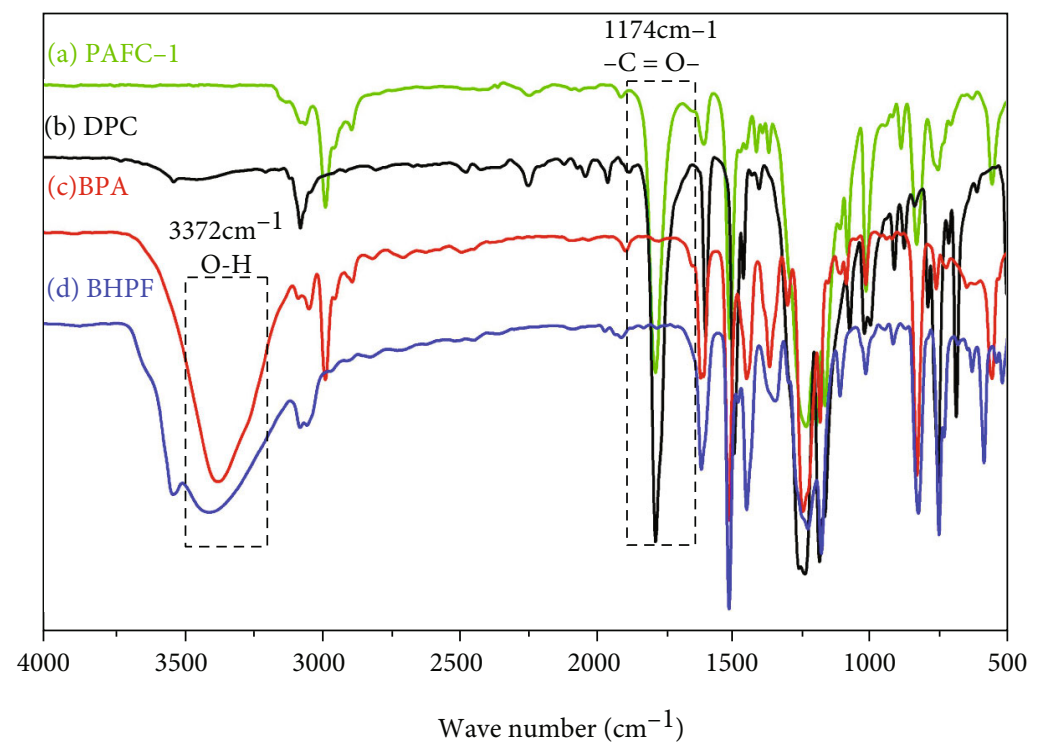

Figure 1: FTIR spectra of BPA, BHPF, DPC, and PAFC-1.

are important to expand their applications [20, 21]. Bales [22] using BHPF with 50\% of the mole ratio to prepare APC improved the impact properties of polycarbonate, solvent resistance, and optical clarity, but also increase its $T_{g}$ up to $270^{\circ} \mathrm{C}$. Toshimasa Tokudu [23] prepared polycarbonate with different molar ratios of BPA and BHPF mixed monomers in order to obtain the vitrification temperature for different applications. Their results showed that when the molar ratio of BPA: BHPF was 30: 70, the product $T_{g}$ was $216^{\circ} \mathrm{C}$, while when the molar ratio of monomer was 15: 85 , the product $T_{g}$ raised to $232^{\circ} \mathrm{C}$. However, considering the high cost of BHPF, it is of significance to develop a method that effectively improves the thermal performance of PC, but with little increase in cost. We prepared a series of polymers with different feed ratios of BPA and BHPF, named PAFC. The BPA/BHPF $(\mathrm{mol} / \mathrm{mol})$ ratios of PAFC1, PAFC-2, PAFC-3, PAFC-4, and PAFC-5 were 99/1, 98/ $2,97 / 3,96 / 4$, and $95 / 5$, respectively. As expected, the thermal performance $\left(T_{g}, T_{g}=203^{\circ} \mathrm{C}\right)$ of PC could be considerably improved by adding only $1 \% \mathrm{BHPF}$, which was about $60^{\circ} \mathrm{C}$ higher than the glass transition temperature of commercial BPA-based PC, thus, potential application value.

\section{Experimental Section}

2.1. Materials. Bisphenol-A (BPA, 99.0\%), fluorene-9bisphenol (BHPF, 99.0\%), and diphenyl carbonate (DPC, 99.0\%) were provided by Aladdin. Sodium bicarbonate $\left(\mathrm{NaHCO}_{3}, 99.5 \%\right)$, zinc acetate $\left(\mathrm{Zn}-(\mathrm{OAc})_{2}, 99.0 \%\right)$, and sodium hydroxide $(\mathrm{NaOH}, 99.0 \%)$ were bought from Tian- jin Tianli. Dichloromethane $\left(\mathrm{CHCl}_{2}, 99.5 \%\right)$, chloroform $\left(\mathrm{CHCl}_{3}, 99.0 \%\right)$, methanol $\left(\mathrm{CH}_{3} \mathrm{OH}, 99.50 \%\right)$, and acetone $\left(\mathrm{CH}_{3} \mathrm{COCH}_{3}, 99.50 \%\right)$ were purchased from Liaoning Quanrui.

2.2. Preparation of Fluorene-9-Bisphenol Polycarbonates. The reaction was carried out in a $100 \mathrm{~mL}$ reaction still. As shown in Scheme 1, the reaction device diagram is shown in Figure S1. A typical polymerization procedure of PAFC (take PAFC-5 for example) is described below. PAFC-5 was prepared by melting transesterification using BPA (10.83 g, $47.5 \mathrm{mmol}), \mathrm{BHPF}(0.875 \mathrm{~g}, 2.5 \mathrm{mmol})$, and DPC $(11.235 \mathrm{~g}, 52.5 \mathrm{mmol})$ as reaction monomers. $\mathrm{NaOH}(1 \mathrm{mg})$ was used as catalyst. Transesterification is the first stage of the reaction. The reactants and catalyst were slowly heated at $160^{\circ} \mathrm{C}$ under a nitrogen atmosphere with stirring and remained for $20 \mathrm{~min}$. Then, the temperature was gradually raised to $180^{\circ} \mathrm{C}$ and remained for another $20 \mathrm{~min}$. Raising the temperature to $200^{\circ} \mathrm{C}$, phenol began to appear at a condenser tube. The reaction temperature was then further increased to $220^{\circ} \mathrm{C}$ for $20 \mathrm{~min}$, to distill phenol off, the byproduct. Condensation polymerization is the second stage of the reaction. After the transesterification process, the temperature of the system was set at $230^{\circ} \mathrm{C}$ under vacuum pressure (200 torr). After $20 \mathrm{~min}$, the temperature was increased to $250^{\circ} \mathrm{C}$ under the vacuum pressure of 150 torr. After further reaction for $20 \mathrm{~min}$, the temperature was increased to $280^{\circ} \mathrm{C}$ under the vacuum pressure of $<0.5$ torr, and the reaction was completed within $20 \mathrm{~min}$. After the polymerization, to obtain PAFC-5, the product was dissolved in dichloromethane and precipitated in 


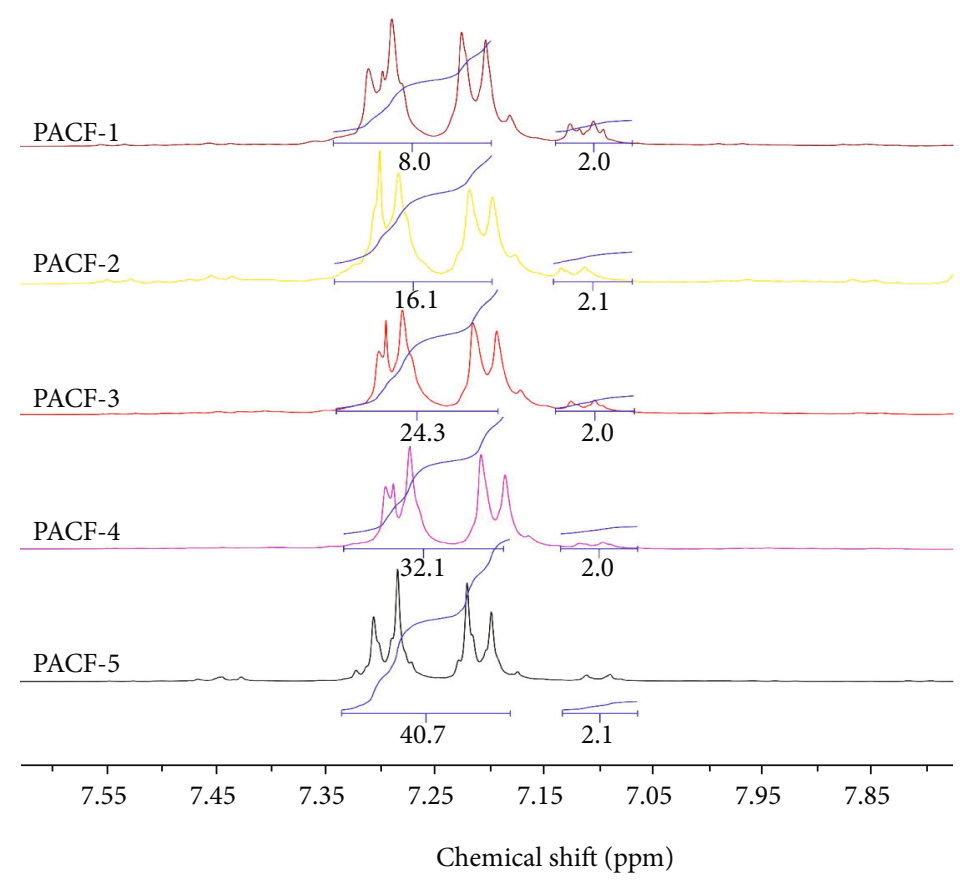

Figure 2: ${ }^{1} \mathrm{H}$ NMR spectra of PAFCs.

methanol and then dried in a vacuum oven. The purified products were used for the characterization of structure and property.

2.3. Analysis of $\triangle C$. The color difference $(\Delta C)$ of the synthesized PAFCs was measured by a UV spectrophotometer (Youke Instrument). This parameter represents the yellowness of the synthesized PAFC. A chloroform solution with a PAFC concentration of $0.01 \mathrm{~g} / \mathrm{mL}$ was used, and $\Delta C$ was calculated as follows:

$$
\Delta C=\left(\left|\frac{1}{3}-\frac{T 445}{T}\right|+\left|\frac{1}{3}-\frac{T 555}{T}\right|+\left|\frac{1}{3}-\frac{T 600}{T}\right|\right) \times 100
$$

where $T_{445}, T_{555}$, and $T_{600}$ represent the transmittance of the polycarbonate solution at a wavelength of 445,555 , and $600 \mathrm{~nm}$, which are relative to chloroform and $T=T_{445}+$ $T_{555}+T_{600}$.

\section{Instruments}

Molecular weight and molecular weight distribution of the polycarbonates were measured on a PL-220 instrument at $40^{\circ} \mathrm{C}$, and tetrahydrofuran was employed as the eluent at a flow rate of $1.0 \mathrm{~mL} / \mathrm{min}$; data were processed using narrow polystyrene standards. Differential scanning calorimetry (DSC) analysis was conducted with alumina 70 ul at a heating or cooling rate of $10^{\circ} \mathrm{C} / \mathrm{min}$, and DSC curves were recorded at the second heating scan from $45^{\circ} \mathrm{C}$ to $600^{\circ} \mathrm{C}$. Rheological property test of copolymer: the prepared copolymer was dried at $120^{\circ} \mathrm{C}$ for $12 \mathrm{~h}$, and its rheological property was tested by hr2 (TA) rheometer. The scanning frequency was $0.05-100 \mathrm{rad} / \mathrm{s}$, and the experimental temperature was $320^{\circ} \mathrm{C}$.
TABLE 1: List of the proton NMR signal.

\begin{tabular}{lccc}
\hline Sample & $\begin{array}{c}\text { BPA/BHPF feed ratio } \\
(\mathrm{mol} / \mathrm{mol})\end{array}$ & \multicolumn{2}{c}{ NMR signal (chemical sift, } \\
ppm)
\end{tabular}

\section{Results and Discussion}

4.1. FT-IR of PAFC. Figure 1 shows the IR spectra of BPA, BHPF, DPC, and PAFC-1 as a typical example. The characteristic absorption peak is about $1769 \mathrm{~cm}^{-1}$, which is attributed to $\mathrm{C}=\mathrm{O}$. In addition, the broad peak at $3000-3600 \mathrm{~cm}^{-}$ ${ }^{1}$ is usually attributed to $\mathrm{O}-\mathrm{H}$ stretching vibration. This broad peak is presumably due to the influence of hydrogen bond on $\mathrm{O}-\mathrm{H}$ in monomers [24]. While the O-H stretching peak of BPA and BHPF at $3000-3600 \mathrm{~cm}^{-1}$ disappears on the infrared spectrum curve of PAFC-1, indicating the occurrence of melt transesterification reaction. PAFC was synthesized successfully.

4.2. ${ }^{1} H$ NMR of PAFCs. In the experiment, NMR tests were carried out on the copolymers of different proportions. The ${ }^{1} \mathrm{H}$ NMR spectra were shown in Figure 2, and the results were summarized in Table 1 . At the chemical shift $\delta=7.07$ 
TABLE 2: Results of copolycondensation of BPA and BHPF with DPC.

\begin{tabular}{|c|c|c|c|c|c|c|c|c|}
\hline Sample & BPA/BHPF feed ratio $(\mathrm{mol} / \mathrm{mol})$ & Yield (\%) & $M_{n}^{\mathrm{a}}(\mathrm{g} / \mathrm{mol})$ & $M_{w}^{\mathrm{a}}(\mathrm{g} / \mathrm{mol})$ & $\mathrm{PDI}^{\mathrm{a}}$ & $\begin{array}{l}T_{g}{ }^{\mathrm{b}} \\
\left({ }^{\circ} \mathrm{C}\right)\end{array}$ & $\begin{array}{c}T_{d-5 \%}{ }^{\mathrm{c}} \\
\left({ }^{\circ} \mathrm{C}\right)\end{array}$ & $\begin{array}{c}\triangle C^{\mathrm{d}} \\
(\%)\end{array}$ \\
\hline PAFC-1 & $99 / 1$ & 92 & 20,900 & 65,000 & 3.10 & 203 & 420 & 1.68 \\
\hline PAFC-2 & $98 / 2$ & 93 & 21,000 & 78,000 & 3.30 & 205 & 425 & 1.70 \\
\hline PAFC-3 & $97 / 3$ & 97 & 23,100 & 105,000 & 3.56 & 207 & 425 & 1.75 \\
\hline PAFC-4 & $96 / 4$ & 98 & 24,800 & 107,000 & 3.31 & 210 & 425 & 1.76 \\
\hline PAFC-5 & $95 / 5$ & 95 & 28,900 & 103,000 & 3.60 & 210 & 425 & 1.85 \\
\hline $\mathrm{APC}^{\mathrm{e}}$ & $100 / 0$ & - & - & - & - & 140 & 320 & - \\
\hline
\end{tabular}

${ }^{\mathrm{a}}$ Determined by THF-GPC. ${ }^{\mathrm{b}}$ Measured by DSC with a heating rate of $10^{\circ} \mathrm{C} \mathrm{min}^{-1}$. ${ }^{\mathrm{c}}$ Degradation temperature for $5 \%$ weight loss was measured by TGA with a heating rate of $10^{\circ} \mathrm{C} \mathrm{m^{-1 }}$ (with $\mathrm{N}_{2}$ ). ${ }^{\mathrm{d}} \triangle \mathrm{C}$ is calculated by formula (1). ${ }^{\mathrm{e}}$ Parameters of conventional polycarbonate.

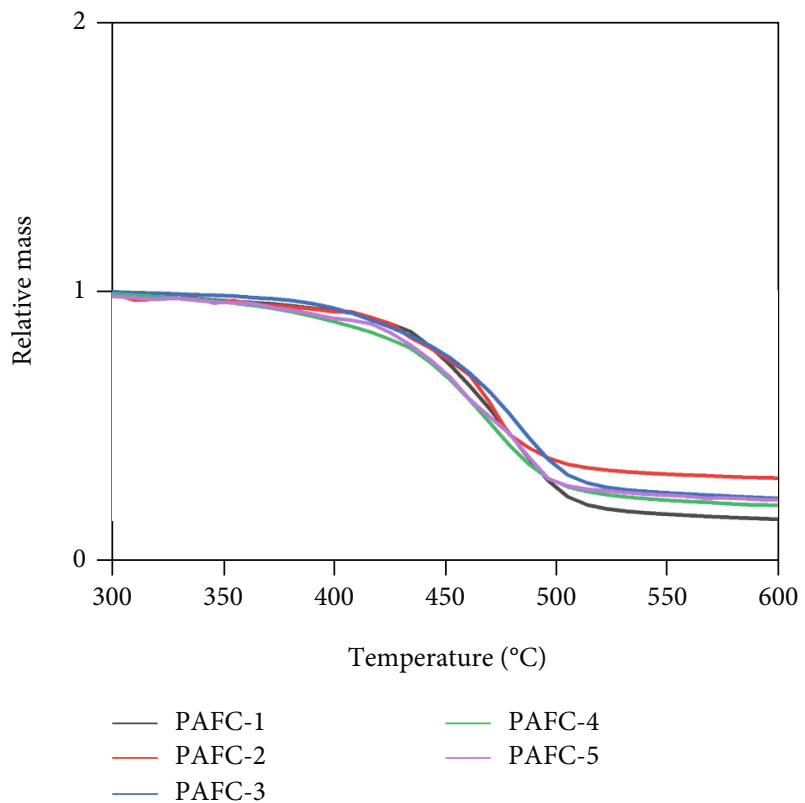

FIgURe 3: TGA curves of PAFCs.

(7.08) - -7.13, the corresponding Ar- $\mathrm{H}$ is on cyclohexane. The chemical shift $\delta=7.18--7.33$ corresponds to $H$ at the BHPF position. Therefore, according to the results of NMR determination, the structure of BPA and BHPF can be preliminarily determined. The number of $H$ at the position of BHPF is proportional, so the content of BHPF was almost consistent with the feed ratios.

4.3. Molecular Weights and Solubility of PAFCs. As summarized in Table 2, Figure 3, GPC data of the copolycarbonate were given. The yield of polycarbonate was all above $90 \%$, with high molecular weights. $\mathrm{Mn}$ and $\mathrm{Mw}$ were in the range of $20,900-28,900 \mathrm{~g} / \mathrm{mol}$ and $65,000-107,000 \mathrm{~g} / \mathrm{mol}$, respectively. The molecular weight distribution of the copolycarbonate was narrow with $\mathrm{Mw} / \mathrm{Mn}$ of 3.1-4.6, which could be attributed to existence of Cardo ring and its symmetrical structure. Meanwhile, the melting transesterification was improved by removing phenol, which increased the polymerization degree of copolycarbonate, thus, high molecular weight of polymers. The process of reducing the yellowness in polymerization is an important issue for PC applied in the field of optical transparent materials. As listed in

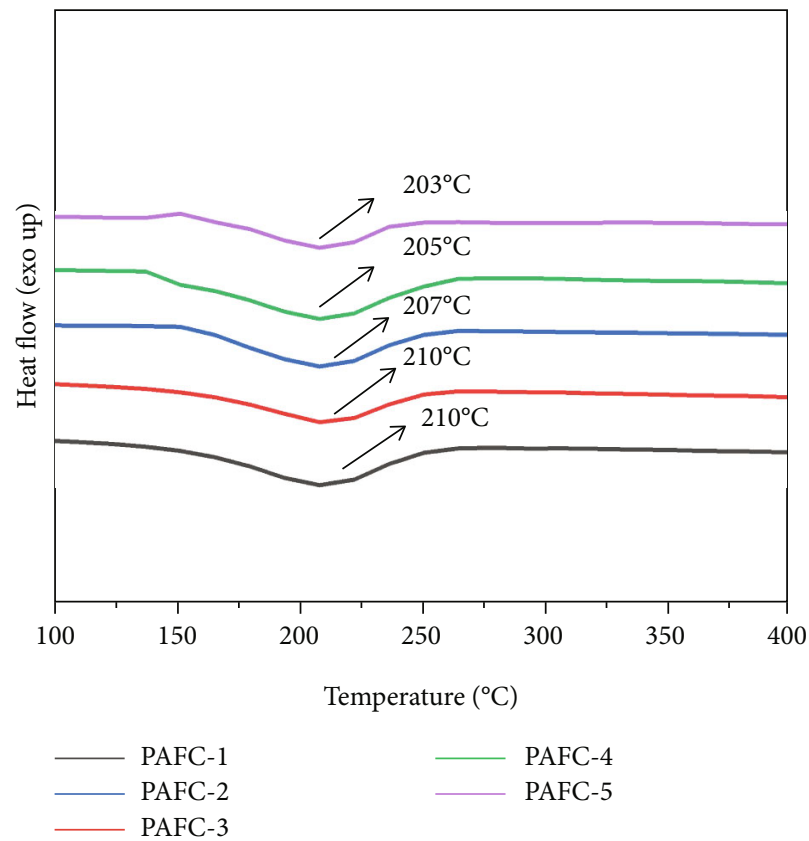

FIgURE 4: DSC curves of PAFCs.

Table 2, the $\triangle C$ of PAFCs is between 1 and 2. The change of $\Delta C$ should mainly be ascribed to the derivation of monomers. Meanwhile, we obtained the copolymer film (Figure S3), and it showed good transparency. A powder $\mathrm{X}$-ray diffraction pattern of the polycarbonate showed the copolycarbonate is amorphous state (Figure S2). The crystal material has poor transparency due to its anisotropy, while the amorphous material has better transparency.

4.4. TGA Curves of PAFCs. Thermal stability is a key property in the polymer processing. Figure 3 shows TGA curves for PAFC-1, PAFC-2, PAFC-3, PAFC-4, and PAFC-5. The temperature at which $5 \mathrm{wt} \%$ is degraded $\left(T_{d-5 \%}\right)$ is given in Table 1. The $T_{d-5 \%}$ date of the copolycarbonates showed that all the PAFC samples were thermally stable up to 420 $425^{\circ} \mathrm{C}$. BHPF has four benzene rings attached to a carbon, and the rigidity of the ring structure is significantly higher than that of a nonring structure. The introduction of monomer in the structure effectively improves the thermal stability of the polymer. The $T_{d}$ of copolymer polycarbonate is 


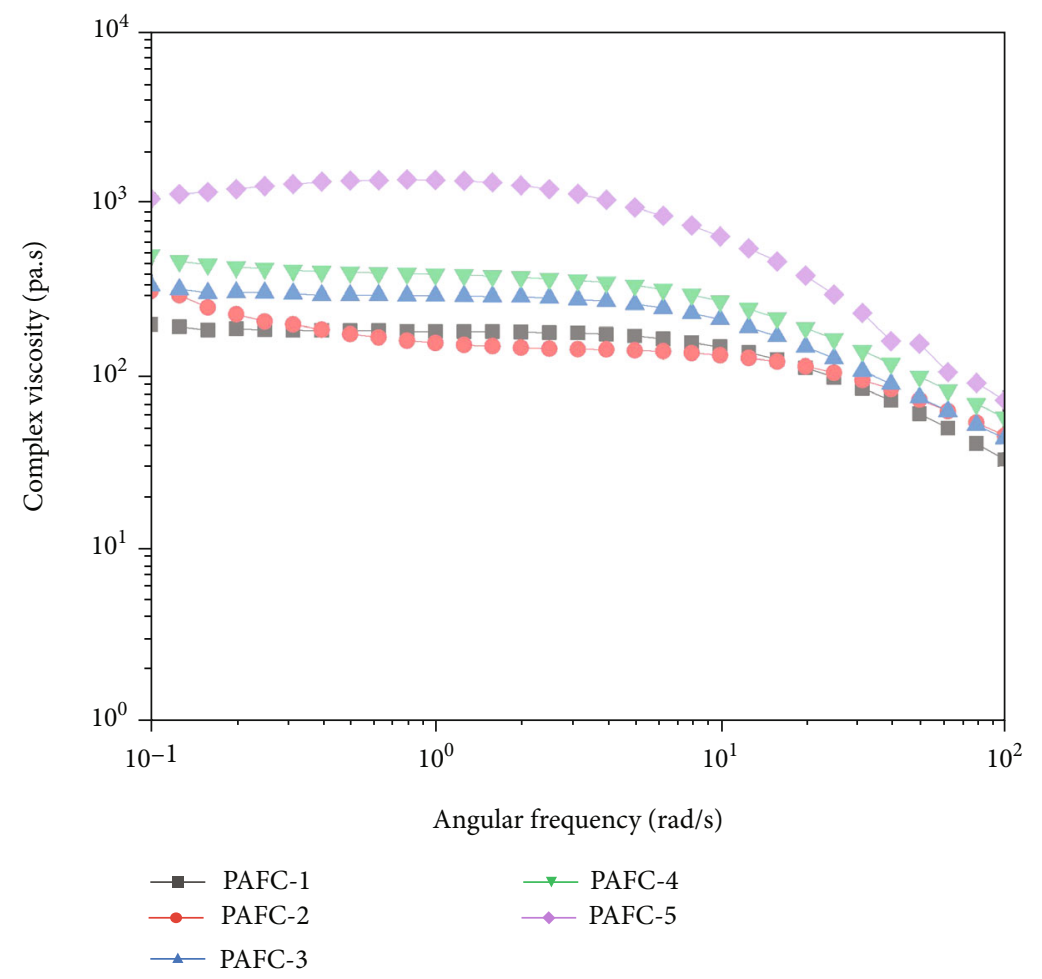

FIGURE 5: Changes of complex viscosity, $\eta$ as function of angular frequency, $\omega$ for $\mathrm{BHPF} /(\mathrm{BPA}+\mathrm{BHPF})$ molar ratio of $\mathrm{PAFC}$ at $320^{\circ} \mathrm{C}$.

function of the content of bisphenol fluorene, going up with the increase of bisphenol fluorene content, own to the thermal stability of bisphenol fluorene. The copolycarbonates we prepared showed a $T_{d-5 \%}$ at $60-75^{\circ} \mathrm{C}$ higher than the control.

4.5. DSC Curves of PAFCs. The $T_{g}$ of the copolycarbonates analyzed using DSC (Figure 4) was 203, 205, 207, 210, and $210^{\circ} \mathrm{C}$, as the content of BHPF was increased from 1 to 5 mol\%. The most important factor affecting $T_{g}$ of copolycarbonates is the flexibility of the molecular chain. The higher the flexibility of the main chain, the lower the $T_{g}$; the higher the rigidity of the main chain, the higher the $T_{g}$ . Thanks to the good thermal stability of Cardo rings in BHPF, the $T_{g}$ of copolycarbonates containing BHPF we prepared was improved considerably.

4.6. The Rheological Curve of PAFCs. Figure 5 displays the viscosity of PAFC-1, PAFC-2, PAFC-3, PAFC-4, and PAFC-5 along with the change of shear velocity under low shear rate. The viscosity change with shear rate almost remains the same. When the copolymer is applied by force, viscous flow performance, and show that the elastic deformation, this is called the rheology of polymer fluid. In Figure 5, in the low shear region, due to the timely reconstruction of the tangles destroyed by shear, the density of tangles remains unchanged, so the viscosity remains unchanged. It is the first Newton region for PAFCs. In the medium shear zone, the tangles are destroyed faster than the reconstruction speed, and the viscosity decreases. That is the pseudoplastic zone for our copolymer. Figure 5 indicates that PAFCs have good processing properties.

\section{Conclusions}

In this study, high-temperature resistant polycarbonates with different BHPF contents were successfully prepared by a melt-polycondensation method with BPA, DPC, and BHPF. The Mw of the prepared PAFC was $107,000 \mathrm{~g} / \mathrm{mol}$, and the thermal properties were greatly improved. The $T_{g}$ of PAFC reached up to $210^{\circ} \mathrm{C}$, which was 1.5 -fold higher than the commercial BPA-based PC $\left(140^{\circ} \mathrm{C}\right) . T_{d-5 \%}$ was as high as $425^{\circ} \mathrm{C}$, which is 1.33 -fold higher than that of commercial BPA-based PC $\left(320^{\circ} \mathrm{C}\right)$. From the experimental results, it can be found that when $1 \mathrm{~mol} \%$ of BHPF was added, the thermal properties $\left(T_{g}\right)$ of polycarbonate are greatly improved. With the content of BHPF was increased from 1 to $5 \mathrm{~mol} \%, T_{g}$ shows a slow-growth trend. This discovery has tremendous application potential in hightemperature resistant plastic industry.

\section{Data Availability}

The data used to support the findings of this study are included within the article and the supplementary information file.

\section{Conflicts of Interest}

The authors declare that they have no conflicts of interest. 


\section{Authors' Contributions}

X.Z. contributed to the conception of the study; Y.L. performed the data analyses and wrote the manuscript; J.W, X.G, X. Li, and X. Liu helped perform the analysis with constructive discussions.

\section{Acknowledgments}

Financial and facility support for this research came from the Fundamental Research Funds in Heilongjiang Provincial universities (CLKFKT2021Z1).

\section{Supplementary Materials}

The following are submitted in "Supporting Files," Figure S1: the reaction device. Figure S2: XRD pattern of the polycarbonate. Figure S3: the picture of the polycarbonate film. (Supplementary Materials)

\section{References}

[1] S. M. Gross, G. W. Roberts, D. J. Kiserow, and J. M. DeSimone, "Synthesis of high molecular weight polycarbonate by solidstate polymerization," Macromolecules, vol. 34, no. 12, pp. 3916-3920, 2001.

[2] S. M. Gross, G. W. Roberts, D. J. Kiserow, and J. M. DeSimone, "Crystallization and solid-state polymerization of poly (bisphenol A carbonate) facilitated by supercritical $\mathrm{CO}_{2}$," Macromolecules, vol. 33, no. 1, pp. 40-45, 2000.

[3] G. H. Choi, D. Y. Hwang, and D. H. Suh, "High thermal stability of bio-based polycarbonates containing cyclic ketal moieties," Macromolecules, vol. 48, no. 19, pp. 6839-6845, 2015.

[4] J. Kim, L. B. Dong, D. J. Kiserow, and G. W. Roberts, “Complex effects of the sweep fluid on solid-state polymerization: poly (bisphenol A carbonate) in supercritical carbon dioxide," Macromolecules, vol. 42, no. 7, pp. 2472-2479, 2009.

[5] M. Zhang, W. Lai, L. Su, and G. Wu, "Effect of catalyst on the molecular structure and thermal properties of isosorbide polycarbonates," Industrial \& Engineering Chemistry Research, vol. 57, no. 14, pp. 4824-4831, 2018.

[6] D. G. Legrand and J. T. Bendler, Handbook of Polycarbonate Science and Technology, Marcel Dekker, New York, 1999.

[7] J. E. Biles, T. P. McNeal, T. H. Begley, and H. C. Hollifield, "Determination of bisphenol A in reusable polycarbonate food-contact plastics and migration to foodsimulating liquids," Journal of Agricultural and Food Chemistry, vol. 45, no. 9, pp. 3541-3544, 1997.

[8] E. J. Hoekstra and C. Simoneau, "Release of bisphenol A from polycarbonate: a review," Critical Reviews in Food Science and Nutrition, vol. 53, no. 4, pp. 386-402, 2013.

[9] S. A. Park, J. Choi, S. Ju et al., "Copolycarbonates of bio-based rigid isosorbide and flexible 1,4-cyclohexanedimethanol: merits over bisphenol-A based polycarbonates," Polymer, vol. 116, pp. 153-159, 2017.

[10] A. Kausar, "A review of filled and pristine polycarbonate blends and their applications," Journal of Plastic Film \& Sheeting, vol. 34, no. 1, pp. 60-97, 2018.

[11] R. R. Shaikh, S. Pornpraprom, and V. D’Elia, “Catalytic strategies for the cycloaddition of pure, diluted, and waste CO2to epoxides under ambient conditions," ACS Catalysis, vol. 8, no. 1, pp. 419-450, 2018.

[12] N. Yadav, F. Seidi, D. Crespy, and V. D'Elia, "Polymers based on cyclic carbonates asTrait d'UnionBetween polymer chemistry and sustainable CO2Utilization," ChemSusChem, vol. 12, no. 4, pp. 724-754, 2019.

[13] M. North, R. Pasquale, and C. Young, "Synthesis of cyclic carbonates from epoxides and CO2," Green Chemistry, vol. 12, no. 9, p. 1514, 2010.

[14] W. Mo, C. Zhuo, H. Cao, S. Liu, X. Wang, and F. Wang, "Facile aluminum porphyrin complexes enable flexible terminal epoxides to boost properties of CO2-polycarbonate," Macromolecular Chemistry and Physics, 2021.

[15] J. Zhang, L. Wang, S. Liu, and Z. Li, "Synthesis of diverse polycarbonates by organocatalytic copolymerization of CO2and epoxides: from high pressure and temperature to ambient conditions," Angewandte Chemie International Edition, vol. 61, no. 4, article e202111197, 2021.

[16] S. Fukuoka, M. Kawamura, K. Komiya et al., "A novel nonphosgene polycarbonate production process using byproduct CO2 as starting material," Green Chemistry, vol. 5, no. 5, pp. 497-507, 2003.

[17] C. Liu, T. Liang, and S. Dongmei, "Application of fluorenone in synthesis of functional polymers," Chemistry and Bonding, vol. 3, pp. 134-136, 2003.

[18] T. Fujimori and M. Hirata, "Polycarbonate resin and process for producing the same," US Patent 6,355,768, 2002.

[19] P. W. Morgan, "Aromatic polyesters with large cross-planar substituents," Macromolecules, vol. 3, no. 5, pp. 536-544, 1970.

[20] C. S. Chen, B. J. Bulkin, and E. M. Pearee, "New epoxy resins. I. The stability of epoxy-trialkoxyboroxines triaryloxyboroxine system," Journal of Applied Polymer Science, vol. 27, no. 4, pp. 1177-1190, 1982.

[21] T. Fujimori and M. Hirata, "Polycarbonate reisn and process for producing the same," US Patent 6,355,768, 2002.

[22] S. E. Bales, J. P. Godschalx, P. C. Yang, M. T. Bishop, and M. J. Marks, "Crosslinkable carbonate Polymers of Dihydroxyaryl fluorine,” US Patent 5,516,877, 1996.

[23] K. Ikeda and T. Tokuda, "Polycarbonate copolymer and heat resistant parts comprising the same," US Patent 7,3170,67, 2008.

[24] N. Maity, S. Barman, Y. Minenkov et al., "A silica-supported monoalkylated tungsten dioxo complex catalyst for olefin metathesis," ACS Catalysis, vol. 8, no. 4, pp. 2715-2729, 2018. 